\title{
A Live Avirulent Mutant Legionella pneumophila Vaccine Induces Protective Immunity against Lethal Aerosol Challenge
}

\author{
Steven J. Blander, Robert F. Breiman, and Marcus A. Horwitz \\ Division of Infectious Diseases, Department of Medicine, University of California, Los Angeles School of Medicine, \\ Los Angeles, California 90024
}

\begin{abstract}
We have examined the capacity of a live avirulent mutant form of the intracellular bacterial pathogen Legionella pneumophila to induce immune responses and protective immunity in guinea pigs. The mutant $L$. pneumophila is nonlethal to guinea pigs and does not revert to virulence with passage through guinea pigs. In contrast, exposure of guinea pigs to aerosols containing wild-type $L$. pneumophila induces a pneumonic illness that clinically and pathologically resembles Legionnaires' disease in humans.
\end{abstract}

Guinea pigs immunized by aerosol exposure to mutant $L$. pneumophila developed a strong humoral immune response to wild-type $L$. pneumophila antigens with reciprocal antibody titers of 32-512 (median 256) by the indirect fluorescent antibody assay, compared with titers of $<2$ for control (sham immunized) guinea pigs. Mutant immunized but not control guinea pigs also developed strong cell-mediated immune responses to wild-type $L$. pneumophila antigens, as demonstrated in assays of cutaneous delayed-type hypersensitivity and in vitro splenic lymphocyte proliferation.

Mutant immunized guinea pigs developed strong protective immunity to lethal aerosol challenge with wild-type $L$. pneumophila. In four independent experiments $40-83 \%$ of mutant immunized guinea pigs survived compared with $0 \%$ of control guinea pigs. Overall, 13 of 21 (62\%) mutant immunized guinea pigs survived compared with 0 of $21(0 \%)$ control guinea pigs $(P=0.00002$, Fisher's exact test, two-tailed). Mutant immunization induced protection comparable to wild-type immunization in these studies; 8 of $14(57 \%)$ guinea pigs immunized by wild-type $L$. pneumophila survived.

This study demonstrates that guinea pigs immunized with a live avirulent mutant $L$. pneumophila vaccine (a) develop a strong humoral immune response to wild-type $L$. pneumophila antigens; (b) develop a strong cell-mediated immune response to wild-type $L$. pneumophila antigens; and (c) develop protective immunity to lethal aerosol challenge with wild-type $L$. pneumophila. This study demonstrates the feasibility of a vaccine against Legionnaires' disease.

Address correspondence to Dr. Marcus A. Horwitz, Division of Infectious Diseases, Department of Medicine, UCLA School of Medicine, Center for the Health Sciences, Room 37-121, Los Angeles, CA 90024.

Received for publication 15 June 1988 and in revised form 21 October 1988

J. Clin. Invest.

(C) The American Society for Clinical Investigation, Inc.

0021-9738/89/03/0810/06 \$2.00

Volume 83, March 1989, 810-815

\section{Introduction}

Legionella pneumophila is a facultative intracellular bacterial pathogen and the causative agent of Legionnaires' disease, a serious and often fatal form of pneumonia (1). Patients with Legionnaires' disease develop both humoral and cell-mediated immune responses to $L$. pneumophila (2-4). Cell-mediated immunity appears to play a primary role in host defense, as activated human monocytes and alveolar macrophages inhibit the intracellular multiplication of L. pneumophila (4-6). Humoral immunity appears to play a secondary role in host defense $(2,3)$.

The guinea pig is an excellent animal model for the study of Legionnaires' disease. When exposed to aerosols containing L. pneumophila guinea pigs develop a pneumonic illness that is clinically and pathologically similar to Legionnaires' disease in humans (7-11). A previous study from this laboratory demonstrated that guinea pigs immunized by a sublethal dose of aerosolized $L$. pneumophila develop humoral and cell-mediated immune responses and protective immunity to subsequent challenge with a lethal aerosol dose of $L$. pneumophila (12).

Another study from this laboratory described an avirulent mutant form of $L$. pneumophila that survives but does not multiply in human monocytes (13). Like the wild-type, the mutant enters monocytes by coiling phagocytosis $(13,14)$. Thereafter, however, the mutant follows an intracellular pathway that differs from the wild-type. Whereas the wild-type phagosome interacts sequentially with host cell smooth vesicles, mitochondria, and ribosomes, a process culminating in the formation of a ribosome-lined replicative phagosome, the mutant phagosome does not interact with these host cell organelles (13). Moreover, whereas the wild-type phagosome does not fuse with lysosomes, the mutant phagosome does fuse with lysosomes so that the mutant comes to reside in a phagolysosome (13). In all other aspects examined the mutant resembles the wild-type. Both organisms are resistant to complement in the presence or absence of anti-L. pneumophila antibody. Both organisms have similar colony morphology, ultrastructure, and structural and secretory protein profiles and lipopolysaccharide profiles on analysis by PAGE.

In the present study we have investigated guinea pig immunologic responses to the mutant form of L. pneumophila, which we have found is nonlethal to guinea pigs. We shall demonstrate that guinea pigs immunized with mutant $L$. pneumophila (a) develop humoral and cell-mediated immune responses to wild-type $L$. pneumophila antigens, and $(b)$ develop protective immunity to lethal aerosol challenge with wild-type L. pneumophila. 


\section{Methods}

Media. RPMI 1640 with L-glutamine was obtained from Gibco Laboratories, Grand Island, NY. Egg yolk buffer $(E Y B)^{1}$ with or without $1 \%$ BSA (1) and PBS (15) were prepared as described previously.

Agar. Modified charcoal yeast extract agar was prepared as described (1).

Bacteria. Wild-type L. pneumophila, Philadelphia 1 strain (serogroup 1), originally obtained from the Center for Disease Control in the homogenate of a spleen of a guinea pig that had died after intraperitoneal inoculation with this strain, was passed one time only in embryonated hens' eggs, harvested, tested for viability, tested for the presence of contaminating bacteria, passed one time only on charcoal yeast extract agar, washed with EYB, flash-frozen in aliquots of $10^{11} \mathrm{CFU} /$ $\mathrm{ml}$, and stored at $-70^{\circ} \mathrm{C}(1)$. Formalin-killed L. pneumophila (FKLP) was prepared as previously described (4). L. pneumophila, mutant 25D (mutant L. pneumophila), obtained and cloned as described (13), was aliquotted and stored in the same way as wild-type L. pneumophila. Before use in the aerosol inoculation system each stock preparation of bacteria was diluted in EYB to the desired concentration.

Membranes. Wild-type L. pneumophila membranes were prepared as described and enumerated in a Petroff-Hausser chamber (16).

Animals. Male Hartley strain guinea pigs weighing $250-300 \mathrm{~g}$, purchased from Charles River Breeding Laboratories (North Wilmington, MA), were housed no more than four to a stainless steel cage and allowed free access to ascorbic acid-fortified chow and water. They were observed in the vivarium for signs of illness for $1 \mathrm{wk}$ before each experiment to ensure that they were healthy. After they were exposed to aerosols of bacteria the guinea pigs were kept in filter-top cages for 1 wk and observed for signs of illness. Their rectal temperatures were taken with a telethermometer with a rectal probe (Yellow Springs Instrument $\mathrm{Co}$., Yellow Springs, $\mathrm{OH}$ ) and their weights were recorded daily.

Immunization of guinea pigs. Guinea pigs were immunized by exposure to aerosols of bacteria in the same lucite aerosol chamber described in detail in a previous study (12). Briefly, the chamber had sealable portals for introducing or removing guinea pigs, an aerosol inlet at the chamber ceiling, and an aerosol nebulizer system (Dart Industries, Ocala, FL). A vacuum pump (Gast Mfg. Co., Benton Harbor, MI) delivered positive pressure to the nebulizer and negative pressure to the chamber. Guinea pigs were immunized by exposure for $30 \mathrm{~min}$ to an aerosol generated from a $10-\mathrm{ml}$ suspension containing a sublethal dose of $2.5 \times 10^{6} \mathrm{CFU}$ of wild-type $L$. pneumophila/ml (wild-type immunized) or $5 \times 10^{9} \mathrm{CFU}$ of mutant L. pneumophila/ml (mutant immunized). Control guinea pigs were sham immunized by exposure for $30 \mathrm{~min}$ to aerosols generated from $10 \mathrm{ml}$ of EYB. After the pump to the nebulizer was shut off the guinea pigs were held inside the chamber for an additional $10 \mathrm{~min}$ before being removed and placed in filter-top cages, three to a cage. Guinea pigs shared cages only with animals exposed to the same type of aerosol. In some experiments guinea pigs were immunized twice, 3 wk apart.

Guinea pig passage. Mutant $L$. pneumophila were passaged three times through guinea pigs to assess their capacity to revert to virulence. On each passage three guinea pigs were exposed to an aerosol generated from a 10-ml suspension containing $5 \times 10^{9} \mathrm{CFU}$ of mutant $L$. pneumophila $/ \mathrm{ml}$. Two of the guinea pigs were observed for $7 \mathrm{~d}$ for signs of illness including fever, weight loss, or change in feeding habits. The remaining guinea pig was killed $2 \mathrm{~d}$ after aerosol exposure. The right lung was sterilely removed and ground in $10 \mathrm{ml}$ of EYB with a mortar and pestle, and CFU in the lung were determined by plating dilutions of the lung suspension in triplicate on charcoal yeast extract agar as described (12). CFU recovered from the lung were harvested from the agar plates, washed with EYB, counted in a Petroff-Hausser chamber, adjusted to a concentration of $5 \times 10^{9}$ bacterial particles $/ \mathrm{ml}$, and used for the subsequent passage.

1. Abbreviations used in this paper: EYB, egg yolk buffer; FKLP, formalin-killed L. pneumophila (wild-type).
Serology. Anti-L. pneumophila antibody in the serum of guinea pigs immunized once or twice with mutant $L$. pneumophila or in the serum of control guinea pigs was assayed by the indirect fluorescent antibody technique as described for human serum by Wilkinson et al. (15). Antigen-bearing slide wells were prepared by covering each well of a 12-well microscope slide (Cel-Line Associates, Minotola, NJ) with $15 \mu$ l of a suspension containing $2 \times 10^{7} \mathrm{FKLP} / \mathrm{ml}$. The slides were air dried, fixed in acetone for $15 \mathrm{~min}$, and then air-dried again. Serum obtained by cardiac puncture from guinea pigs was serially diluted twofold in PBS up to a concentration of 1:1,024, and $20 \mu$ l of each concentration was applied to each well. The slides were incubated at $37^{\circ} \mathrm{C}$ in $100 \%$ humidity for $30 \mathrm{~min}$, rinsed, and then soaked in PBS. 10 $\mu \mathrm{l}$ of fluorescein-conjugated IgG fraction goat anti-guinea pig IgG (Fc fragment) (Cappel Laboratories, Cochranville, PA) was placed on each well and the slides were then incubated at $37^{\circ} \mathrm{C}$ in $100 \%$ humidity for $30 \mathrm{~min}$ and rinsed with PBS. The slides were magnified 1,000 times with a fluorescence microscope (Nikon Inc., Garden City, NY). The titer of each animal's serum was expressed as the reciprocal of the dilution that produced $2+$ fluorescent staining of at least $50 \%$ of the FKLP in a microscopic field.

Cutaneous delayed-type hypersensitivity. Guinea pigs were shaved over the back and flank and administered $100 \mu \mathrm{l}$ of FKLP diluted in EYB to a concentration of $10^{8}$ or $10^{9}$ bacterial particles $/ \mathrm{ml}$ or $100 \mu \mathrm{l}$ of control buffer (EYB) intradermally at different sites in the shaved area. The diameters of areas of erythema and induration at these sites were recorded at 24,48 , and $72 \mathrm{~h}$ after injection.

Lymphocyte proliferation assay. Splenic lymphocytes from mutant immunized or control guinea pigs were obtained and purified, incubated with antigens (L. pneumophila membranes or FKLP) in triplicate microtest wells for $2 \mathrm{~d}$, and then tested for capacity to incorporate $\left[{ }^{3} \mathrm{H}\right]$ thymidine as described (12).

Studies of protective immunity. Guinea pigs were immunized once or twice by exposure to an aerosol generated from a 10-ml suspension containing $5 \times 10^{9} \mathrm{CFU} / \mathrm{ml}$ of mutant L. pneumophila or $2.5 \times 10^{6}$ $\mathrm{CFU} / \mathrm{ml}$ of wild-type $L$. pneumophila (sublethal dose). Where guinea pigs were immunized twice, the second immunization was $3 \mathrm{wk}$ after the first. Control guinea pigs were sham immunized once or twice. 3 wk after immunization all guinea pigs were challenged with an aerosolized dose of wild-type $L$. pneumophila. The challenge dose of wild-type L. pneumophila $\left(2.5 \times 10^{8} \mathrm{CFU} / \mathrm{ml}\right)$, hereinafter referred to as one lethal dose $\left(\mathrm{LD}_{100}\right)$, was the lowest dose that consistently resulted in the death of all control guinea pigs (12). After the challenge the number of survivors in each group of animals was quantitated. Differences in survival between groups of animals were evaluated by the Fisher's exact test, two-tailed.

\section{Results}

Mutant L. pneumophila are nonlethal to guinea pigs. Guinea pigs were completely resistant to challenge with even very high concentrations of aerosolized mutant $L$. pneumophila. Whereas $100 \%$ of guinea pigs died after exposure to an aerosol generated from a 10-ml suspension containing $2.5 \times 10^{8} \mathrm{CFU}$ of wild-type $L$. pneumophila $/ \mathrm{ml}, 0 \%$ of guinea pigs died after exposure to an aerosol generated from a $10-\mathrm{ml}$ suspension containing a 20 -fold higher concentration $\left(5 \times 10^{9} \mathrm{CFU} / \mathrm{ml}\right)$ of mutant $L$. pneumophila, the highest concentration tested.

Mutant L. pneumophila remain avirulent after passage through guinea pigs. Mutant L. pneumophila did not revert to virulence after three sequential aerosol passages through guinea pigs. During the 7-d observation period after each passage guinea pigs did not become ill. Moreover, in guinea pigs killed $2 \mathrm{~d}$ after passage, CFU of $L$. pneumophila in the lungs did not increase with successive passages. In contrast, in studies not presented here that used the same methodology, three 
Table I. Anti-L. pneumophila Antibody Titers in the Sera of Mutant Immunized and Control Guinea Pigs

\begin{tabular}{|c|c|c|c|c|c|c|c|}
\hline \multicolumn{4}{|c|}{ One immunization } & \multicolumn{4}{|c|}{ Two immunizations } \\
\hline \multirow[b]{2}{*}{ Guinea pig pair } & \multirow[b]{2}{*}{ Time after immunization } & \multicolumn{2}{|c|}{ Reciprocal antibody titer } & \multirow[b]{2}{*}{ Guinea pig pair } & \multirow[b]{2}{*}{$\begin{array}{c}\text { Time after } \\
\text { first immunization }\end{array}$} & \multicolumn{2}{|c|}{ Reciprocal antibody titer } \\
\hline & & $\begin{array}{l}\text { Mutant immunized } \\
\text { guinea pigs }\end{array}$ & $\begin{array}{l}\text { Control } \\
\text { guinea pigs }\end{array}$ & & & $\begin{array}{l}\text { Mutant immunized } \\
\text { guinea pigs }\end{array}$ & $\begin{array}{c}\text { Control } \\
\text { guinea pigs }\end{array}$ \\
\hline & $w k$ & & & & $w k$ & & \\
\hline $\mathbf{A}$ & 3 & 32 & $<2$ & $\mathbf{F}$ & 6 & 512 & $<2$ \\
\hline B & 3 & 64 & $<2$ & $\mathbf{G}$ & 6 & 256 & $<2$ \\
\hline $\mathrm{C}$ & 4 & 256 & $<2$ & $\mathbf{H}$ & 6 & 512 & $<2$ \\
\hline D & 6 & 256 & $<2$ & & & & \\
\hline $\mathbf{E}$ & 6 & 128 & $<2$ & & & & \\
\hline
\end{tabular}

Guinea pigs were immunized with aerosolized mutant $L$. pneumophila or sham immunized (control guinea pigs) once or twice as indicated. Where guinea pigs were immunized twice, the second immunization was $3 \mathbf{w k}$ after the first. At the time after immunization indicated, serum was obtained and the anti-L. pneumophila antibody titer determined by the indirect fluorescent antibody assay as described in the text. The titer was expressed as the reciprocal dilution that produced $2+$ fluorescence of at least $50 \%$ of the FKLP in a microscopic field.

unrelated (noncloned) avirulent strains of Legionellae rapidly regained virulence upon guinea pig passage. With these strains reversion to virulence was apparent by the second passage and $\mathrm{CFU}$ in the lungs of guinea pigs increased dramatically (1-5 logs) with successive passages.
Guinea pigs exposed to aerosolized mutant L. pneumophila develop humoral immune responses to wild-type L. pneumophila antigens. To determine if guinea pigs exposed to aerosolized mutant $L$. pneumophila develop humoral immune responses to wild-type $L$. pneumophila, we measured the anti-

Table II. Skin Reactivity of Mutant Immunized, Wild-type Immunized, and Control Guinea Pigs in Response to Wild-type L. pneumophila Antigens

\begin{tabular}{|c|c|c|c|c|c|c|c|c|c|}
\hline \multirow[b]{3}{*}{ Experiment } & \multirow[b]{3}{*}{ Status of guinea pigs } & \multirow{3}{*}{$\begin{array}{l}\text { Number of } \\
\text { guinea pigs }\end{array}$} & \multirow[b]{3}{*}{ Skin reaction } & \multicolumn{6}{|c|}{$\begin{array}{l}\text { Extent of erythema and induration (millimeters) in response to indicated antigen } \\
\text { concentration (bacterial particles/milliliter) }\end{array}$} \\
\hline & & & & \multicolumn{2}{|c|}{$24 \mathrm{~h}$} & \multicolumn{2}{|c|}{$48 \mathrm{~h}$} & \multicolumn{2}{|c|}{$72 \mathrm{~h}$} \\
\hline & & & & $10^{8}$ & $10^{9}$ & $10^{8}$ & $10^{9}$ & $10^{8}$ & $10^{9}$ \\
\hline \multirow[t]{6}{*}{ A } & Mutant immunized & 4 & Erythema & $8.8 \pm 2.2$ & $9.0 \pm 1.0$ & $8.8 \pm 1.9$ & $12.0 \pm 1.1$ & $4.7 \pm 0.9$ & $9.3 \pm 0.7$ \\
\hline & & & Induration & $6.5 \pm 3.0$ & $8.3 \pm 1.0$ & $5.5 \pm 3.2$ & $10.0 \pm 1.5$ & $2.0 \pm 1.0$ & $6.0 \pm 0.6$ \\
\hline & Control & 4 & Erythema & $8.3 \pm 1.7$ & $8.3 \pm 1.7$ & $4.8 \pm 2.9$ & $8.8 \pm 3.4$ & $0.0 \pm 0.0$ & $5.0 \pm 2.9$ \\
\hline & & & Induration & $7.3 \pm 1.5$ & $7.0 \pm 1.8$ & $2.8 \pm 1.0$ & $6.5 \pm 2.7$ & $0.0 \pm 0.0$ & $2.8 \pm 1.6$ \\
\hline & & & SI (erythema)* & 1.1 & 1.1 & 1.8 & 1.4 & $\infty$ & 1.9 \\
\hline & & & SI (induration) & 0.9 & 1.2 & 2.0 & 1.5 & $\infty$ & 2.1 \\
\hline \multirow[t]{7}{*}{ B } & Mutant immunized & 3 & Erythema & $8.0 \pm 1.3$ & $9.3 \pm 1.5$ & $2.3 \pm 1.5$ & $6.2 \pm 0.4$ & $3.3 \pm 0.9$ & $8.7 \pm 0.7$ \\
\hline & & & Induration & $0.0 \pm 0.0$ & $4.2 \pm 0.4$ & $0.0 \pm 0.0$ & $4.2 \pm 0.4$ & $0.0 \pm 0.0$ & $3.8 \pm 0.4$ \\
\hline & Control & 3 & Erythema & $2.8 \pm 0.4$ & $6.0 \pm 0.6$ & $1.5 \pm 0.9$ & $4.3 \pm 1.2$ & $1.5 \pm 0.5$ & $5.8 \pm 0.6$ \\
\hline & & & Induration & $0.5 \pm 0.5$ & $3.0 \pm 0.0$ & $0.0 \pm 0.0$ & $2.3 \pm 1.2$ & $0.0 \pm 0.0$ & $2.5 \pm 0.5$ \\
\hline & & & SI (erythema) & 2.9 & 1.6 & 1.5 & 1.4 & 2.2 & 1.5 \\
\hline & & & SI (induration) & 0.0 & 1.4 & - & 1.8 & - & 1.5 \\
\hline & & & & $10^{9}$ & $10^{10}$ & $10^{9}$ & $10^{10}$ & $10^{9}$ & $10^{10}$ \\
\hline \multirow[t]{8}{*}{ C } & Mutant immunized & 2 & Erythema & $13.8 \pm 4.3$ & $17.5 \pm 2.5$ & $10.3 \pm 3.3$ & $13.0 \pm 4.0$ & $10.5 \pm 1.5$ & $14.8 \pm 3.3$ \\
\hline & & & Induration & $6.5 \pm 0.5$ & $10.3 \pm 0.3$ & $4.8 \pm 1.8$ & $8.3 \pm 1.3$ & $4.5 \pm 0.5$ & $9.8 \pm 0.3$ \\
\hline & Control & 4 & Erythema & $7.8 \pm 0.8$ & $10.7 \pm 0.8$ & $5.8 \pm 1.5$ & $12.5 \pm 0.9$ & $6.6 \pm 1.1$ & $15.6 \pm 2.1$ \\
\hline & & & Induration & $2.9 \pm 0.7$ & $6.0 \pm 0.4$ & $1.6 \pm 0.9$ & $4.9 \pm 0.4$ & $2.1 \pm 0.6$ & $8.3 \pm 1.2$ \\
\hline & & & SI (erythema) & 1.8 & 1.6 & 1.8 & 1.0 & 1.6 & 0.9 \\
\hline & & & SI (induration) & 2.2 & 1.7 & 3.0 & 1.7 & 2.1 & 1.2 \\
\hline & Wild-type immunized & 2 & Erythema & $15.5 \pm 1.5$ & $18.5 \pm 0.5$ & $14.3 \pm 1.8$ & $21.3 \pm 1.3$ & $15.3 \pm 1.8$ & $24.3 \pm 1.8$ \\
\hline & & & Induration & $8.5 \pm 0.5$ & $12.5 \pm 0.5$ & $6.8 \pm 0.8$ & $11.5 \pm 0.5$ & $7.0 \pm 0.0$ & $11.5 \pm 1.5$ \\
\hline
\end{tabular}

Guinea pigs were immunized with mutant $L$. pneumophila or sham immunized (control) once (experiment A) or twice 3 wk apart (experiments B and C). In experiment $C$ a third group of animals was immunized twice ( 3 wk apart) by sublethal infection with wild-type L. pneumophila (wild-type immunized). All animals were skin tested with FKLP as described in the text. Data given are mean \pm SEM. *SI, mean skin reactivity (millimeters) mutant immunized guinea pigs/mean skin reactivity (millimeters) of control guinea pigs. 
body titer to FKLP in the serum of mutant immunized and control animals by the indirect fluorescent antibody assay. All mutant immunized animals developed significant antibody titers ( $\geq 32$ ); the geometric mean titer for all such animals was 181. In contrast, all control guinea pigs had titers of $<2$ (Table I).

Guinea pigs immunized with mutant L. pneumophila develop cutaneous delayed-type hypersensitivity to wild-type $L$. pneumophila antigens. To determine if mutant immunized guinea pigs develop a cutaneous delayed-type hypersensitivity response to wild-type antigens, we measured the extent of erythema and induration at skin test sites where mutant immunized and control guinea pigs were injected intradermally with various concentrations of FKLP 3 wk after immunization (Table II). Mutant immunized guinea pigs consistently had greater mean areas of erythema and induration than control guinea pigs. In 18 comparisons made between groups of immunized and control guinea pigs in three independent experi- ments (Table II), mutant immunized guinea pigs had greater mean areas of erythema in $17(94 \%)$ comparisons, and greater mean areas of induration in $16(89 \%)$ comparisons. The extent of erythema and induration in immunized guinea pigs was typically about two-fold that in control guinea pigs. The erythema in control animals was distinctly lighter in color than the deep red erythema in the mutant immunized animals. Intradermal injection of the diluting buffer, EYB, did not produce erythema or induration in mutant immunized or control animals.

Splenic lymphocytes from guinea pigs immunized with mutant L. pneumophila proliferate in response to wild-type $L$. pneumophila antigens. To determine if mutant immunized guinea pigs expand a population of lymphocytes that recognize wild-type $L$. pneumophila antigens, we studied the proliferative responses of splenic lymphocytes from these animals to FKLP and to wild-type L. pneumophila membranes (Table III).

Table III. Proliferation of Splenic Lymphocytes from Mutant Immunized and Control Guinea Pigs in Response to Wild-type L. pneumophila Antigens

\begin{tabular}{|c|c|c|c|c|c|c|c|c|c|c|c|}
\hline \multirow[b]{3}{*}{ Experiment } & & \multirow{3}{*}{$\begin{array}{l}\text { Time after } \\
\text { immunization }\end{array}$} & \multirow{3}{*}{$\begin{array}{c}\text { No } \\
\text { antigen }\end{array}$} & \multicolumn{4}{|c|}{ FKLP } & \multicolumn{4}{|c|}{ Membrane } \\
\hline & & & & \multicolumn{2}{|c|}{$5 \times 10^{6} / \mathrm{ml}$} & \multicolumn{2}{|c|}{$5 \times 10^{7} / \mathrm{ml}$} & \multicolumn{2}{|c|}{$5 \times 10^{6} / \mathrm{ml}$} & \multicolumn{2}{|c|}{$5 \times 10^{7} / \mathrm{ml}$} \\
\hline & & & & & $\mathrm{SI}^{*}$ & & SI & & SI & & SI \\
\hline & & $w k$ & $c p m$ & $c p m$ & & $c p m$ & & cpm & & cpm & \\
\hline \multicolumn{12}{|c|}{$\begin{array}{l}\text { One immunization } \\
\text { Immunized guinea pigs }\end{array}$} \\
\hline A & Al & 3 & $142 \pm 4$ & $1,320 \pm 52$ & 9.3 & $2,199 \pm 187$ & 15.5 & $452 \pm 30$ & 3.2 & $1,299 \pm 131$ & 9.1 \\
\hline \multirow[t]{2}{*}{ B } & B1 & 3 & $138 \pm 7$ & $1,337 \pm 36$ & 9.7 & $1,865 \pm 77$ & 13.5 & - & - & $1,368 \pm 229$ & 24.4 \\
\hline & B2 & 6 & $146 \pm 9$ & $1,266 \pm 139$ & 8.7 & $1,748 \pm 44$ & 12.0 & - & - & $1,469 \pm 81$ & 10.1 \\
\hline \multirow[t]{4}{*}{$\mathrm{C}$} & $\mathrm{Cl}$ & 3 & $167 \pm 88$ & $786 \pm 133$ & 4.7 & $3,394 \pm 213$ & 20.3 & $616 \pm 35$ & 3.7 & $4,722 \pm 327$ & 28.3 \\
\hline & $\mathrm{C} 2$ & 4 & $97 \pm 4$ & $239 \pm 2$ & 2.5 & $454 \pm 0$ & 4.7 & $193 \pm 12$ & 2.0 & $523 \pm 26$ & 5.4 \\
\hline & $\mathrm{C} 3$ & 6 & $182 \pm 7$ & $596 \pm 39$ & 3.3 & $2,432 \pm 49$ & 13.4 & $519 \pm 171$ & 2.9 & $3,529 \pm 1,067$ & 19.4 \\
\hline & Mean & & & & $6.4 \pm 1.3$ & & $13.2 \pm 2.1$ & & $3.0 \pm 0.4$ & & $16.1 \pm 3.8$ \\
\hline \multicolumn{12}{|l|}{ Control guinea pigs } \\
\hline A & Al & 3 & $181 \pm 7$ & $412 \pm 47$ & 2.3 & $1,551 \pm 165$ & 8.6 & $203 \pm 3$ & 1.1 & $473 \pm 36$ & 2.6 \\
\hline \multirow[t]{2}{*}{ B } & B1 & 3 & $220 \pm 11$ & $755 \pm 37$ & 3.4 & $1,317 \pm 47$ & 6.0 & - & - & $2,521 \pm 126$ & 11.5 \\
\hline & B2 & 6 & $110 \pm 5$ & $179 \pm 6$ & 1.6 & $308 \pm 33$ & 2.8 & - & - & $535 \pm 29$ & 4.9 \\
\hline \multirow[t]{5}{*}{$\mathrm{C}$} & $\mathrm{Cl}$ & 3 & $121 \pm 7$ & $195 \pm 7$ & 1.6 & $452 \pm 45$ & 3.7 & $218 \pm 1$ & 1.8 & $941 \pm 5$ & 7.8 \\
\hline & $\mathrm{C} 2$ & 4 & $110 \pm 6$ & $157 \pm 55$ & 1.4 & $124 \pm 1$ & 1.1 & $96 \pm 3$ & 0.9 & $211 \pm 29$ & 1.9 \\
\hline & $\mathrm{C} 3$ & 6 & $195 \pm 7$ & $514 \pm 68$ & 2.6 & $989 \pm 145$ & 5.1 & $323 \pm 28$ & 1.7 & $1,840 \pm 381$ & 9.4 \\
\hline & Mean & & & & $2.2 \pm 0.3$ & & $4.6 \pm 1.1$ & & $1.4 \pm 0.2$ & & $6.4 \pm 1.6$ \\
\hline & $P^{\ddagger}<$ & & & & 0.01 & & 0.005 & & 0.005 & & 0.03 \\
\hline \multicolumn{12}{|c|}{$\begin{array}{l}\text { Two immunizations } \\
\text { Immunized guinea pigs }\end{array}$} \\
\hline \multirow[t]{4}{*}{ D } & D1 & 3 & $221 \pm 18$ & $1,187 \pm 27$ & 5.4 & & & $440 \pm 15$ & 2.0 & $1,845 \pm 264$ & 8.3 \\
\hline & D2 & 3 & $109 \pm 4$ & $711 \pm 65$ & 6.5 & & & $288 \pm 32$ & 2.6 & $634 \pm 32$ & 5.8 \\
\hline & D3 & 3 & $113 \pm 7$ & $1,000 \pm 33$ & 8.8 & & & $627 \pm 32$ & 5.5 & $339 \pm 29$ & 3.0 \\
\hline & Mean & & & & $6.9 \pm 1.0$ & & & & $3.4 \pm 1.1$ & & $5.7 \pm 1.5$ \\
\hline \multicolumn{12}{|l|}{ Control guinea pigs } \\
\hline \multirow[t]{5}{*}{ D } & D1 & 3 & $267 \pm 13$ & $634 \pm 1$ & 2.4 & & & $325 \pm 34$ & 1.2 & $550 \pm 89$ & 2.1 \\
\hline & D2 & 3 & $101 \pm 5$ & $133 \pm 26$ & 1.3 & & & $106 \pm 1$ & 1.0 & $223 \pm 15$ & 2.2 \\
\hline & D3 & 3 & $109 \pm 4$ & $109 \pm 3$ & 2.2 & & & $127 \pm 11$ & 1.2 & $188 \pm 20$ & 1.7 \\
\hline & Mean & & & & $2.0 \pm 0.3$ & & & & $1.1 \pm 0.1$ & & $2.0 \pm 0.2$ \\
\hline & $P^{\ddagger}<$ & & & & 0.005 & & & & 0.1 & & 0.05 \\
\hline
\end{tabular}

In each of four independent experiments (A-D), proliferation of splenic lymphocytes from one to three pairs of mutant immunized and control animals was studied. Mutant immunized guinea pigs were exposed to mutant $L$. pneumophila once (experiments A-C) or twice, 3 wk apart (experiment D). Splenic lymphocytes (10 ${ }^{7}$ cells $/ \mathrm{ml})$ were incubated in microtest wells at $37^{\circ} \mathrm{C}$ for $2 \mathrm{~d}$ without antigen or with either FKLP $\left(5 \times 10^{6}\right.$ or $\left.5 \times 10^{7} / \mathrm{ml}\right)$ or wild-type L. pneumophila membranes $(5$ $\times 10^{6}$ or $5 \times 10^{7} / \mathrm{ml}$ ). The lymphocytes were then assayed for their capacity to incorporate $\left[{ }^{3} \mathrm{H}\right]$ thymidine as described in the text. For each animal the mean $\mathrm{cpm} \pm \mathrm{SEM}$ for six wells without antigen or three wells with antigen is reported. $*$ SI, stimulation index $=\left(\mathrm{mean}\left[{ }^{3} \mathrm{H}\right]\right.$ thymidine incorporation (cpm) of lymphocytes incubated with antigen)/(mean $\left[{ }^{3} \mathrm{H}\right]$ thymidine incorporation $(\mathrm{cpm})$ of lymphocytes incubated without antigen). ${ }^{\ddagger} P$ value for difference between SI of immunized and control guinea pigs by two-tailed $t$-test. 
In four experiments involving nine pairs of mutant immunized and control guinea pigs, the mutant immunized animals consistently had significantly greater splenic lymphocyte proliferative responses to $L$. pneumophila antigens than did control animals (Table III). The immunized animals exhibited significantly stronger lymphocyte proliferative responses to both FKLP and L. pneumophila membrane antigens. Stimulation indices of immunized animals were approximately threefold those of control animals in response to either of the $L$. pneumophila antigen preparations. Stimulation indices of guinea pigs immunized with mutant $L$. pneumophila twice were not significantly different from those of guinea pigs immunized once.

Guinea pigs immunized with mutant L. pneumophila are protected against lethal aerosol challenge. We performed four experiments to determine if mutant immunized guinea pigs are protected against lethal aerosol challenge with wild-type $L$. pneumophila (Table IV). Each experiment had a negative sham-immunized control group consisting of animals exposed to aerosolized diluting buffer (EYB), and three of the experiments had a positive control group consisting of animals immunized by sublethal infection with wild-type $L$. pneumophila (wild-type immunized). In the first two experiments animals were immunized once before challenge. In the second two experiments animals were immunized twice 3 wk apart before challenge. Altogether, 21 control animals, 21 mutant immunized animals, and 14 wild-type immunized animals were challenged.

Mutant immunization produced strong protective immunity. Whereas none $(0 \%)$ of the control animals survived chal-

Table IV. Survival Rates of Immunized and Control Guinea Pigs Administered a Lethal Aerosol Challenge of Wild-type L. pneumophila

\begin{tabular}{|c|c|c|c|c|}
\hline \multirow{4}{*}{$\begin{array}{l}\begin{array}{c}\text { Experimental status } \\
\text { of guinea pig }\end{array} \\
\begin{array}{l}\text { One immunization } \\
\text { Control } \\
\text { Mutant } \\
\text { immunized }\end{array}\end{array}$} & \multicolumn{3}{|c|}{$\begin{array}{l}\text { Number of guinea pigs surviving per number } \\
\text { challenged and \% survival }\end{array}$} & \multirow[t]{2}{*}{$P^{*}$} \\
\hline & \multirow{2}{*}{$\begin{array}{l}\text { Experiment } 1 \\
0 / 5(0 \%)\end{array}$} & \multirow{2}{*}{$\begin{array}{c}\text { Experiment } 2 \\
0 / 5(0 \%)\end{array}$} & \multirow{2}{*}{$\begin{array}{c}\text { Total } \\
0 / 10(0 \%)\end{array}$} & \\
\hline & & & & \\
\hline & $3 / 4(75 \%)$ & $2 / 5(40 \%)$ & $5 / 9 \quad(56 \%)$ & 0.01 \\
\hline \multicolumn{5}{|l|}{ Wild-type } \\
\hline immunized & $2 / 5(40 \%)$ & $4 / 5(80 \%)$ & $6 / 10(60 \%)$ & 0.01 \\
\hline Two immunizations & Experiment 3 & Experiment 4 & Total & \\
\hline Control & $0 / 5(0 \%)$ & $0 / 6(0 \%)$ & $0 / 11(0 \%)$ & - \\
\hline \multicolumn{5}{|l|}{ Mutant } \\
\hline immunized & $5 / 6(83 \%)$ & $3 / 6(50 \%)$ & $8 / 12(67 \%)$ & 0.001 \\
\hline \multicolumn{5}{|l|}{ Wild-type } \\
\hline immunized & $2 / 4(50 \%)$ & - & $2 / 4(50 \%)$ & 0.06 \\
\hline \multicolumn{5}{|l|}{ Grand total } \\
\hline Control & & & $0 / 21(0 \%)$ & 一 \\
\hline \multicolumn{5}{|l|}{ Mutant } \\
\hline immunized & & & $13 / 21(62 \%)$ & 0.00002 \\
\hline \multicolumn{5}{|l|}{ Wild-type } \\
\hline immunized & & & $8 / 14(57 \%)$ & 0.0001 \\
\hline
\end{tabular}

In four independent experiments guinea pigs were immunized once or twice ( 3 wk apart) by exposure to mutant $L$. pneumophila or to a sublethal dose of wild-type $L$. pneumophila (three experiments), or they were sham immunized (control guinea pigs). 3 wk after immunization all animals were challenged with a lethal aerosol dose of wild-type $L$. pneumophila and survival was quantitated.

* $P$ value for difference between mutant immunized and control animals or for difference between wild-type immunized and control animals by the Fisher's exact test, two-tailed. lenge in any experiment, $40-83 \%$ of mutant immunized animals survived. Similarly, 40-80\% of wild-type immunized animals survived. Overall, a mean of $62 \%$ of mutant immunized animals and $57 \%$ of wild-type immunized animals survived challenge. The difference in survival between mutant immunized and control animals was highly significant $(P=0.00002$, Fisher's exact test, two-tailed). The difference in overall survival between mutant immunized and wild-type immunized guinea pigs was not statistically significant. Somewhat more animals immunized with mutant $L$. pneumophila twice $(67 \%)$ than animals immunized with mutant $L$. pneumophila once (56\%) survived, but this difference was also not statistically significant.

The mean weights of animals in the three groups were not significantly different $(P>0.05)$ from each other either at the start of the experiment, before administration of any immunizing doses, or on the day of challenge $3 \mathrm{wk}$ after administration of the last immunizing dose. After lethal challenge guinea pigs in all three groups rapidly lost weight. $2 \mathrm{~d}$ after challenge, before animals died, immunized animals lost significantly more weight $(P<0.025)$ than control animals. The mean weight loss for mutant immunized guinea pigs was $47 \pm 7 \mathrm{~g}$, for wild-type immunized animals $44 \pm 17 \mathrm{~g}$, and for control animals $25 \pm 7 \mathrm{~g}$.

After lethal challenge, during the course of illness, control guinea pigs exhibited more frequent and severe signs of illness such as labored respirations and decreased activity than immunized animals. However, a majority of mutant immunized and wild-type immunized animals also exhibited these signs of illness at some point after challenge. Two guinea pigs from each experimental group were observed to have loose stools. Some guinea pigs had elevated temperatures over the 7-d observation period, but there was no obvious relationship between immunization status and febrile response.

\section{Discussion}

We have demonstrated that immunization of guinea pigs with a live avirulent mutant form of $L$. pneumophila induces protective immunity against lethal aerosol challenge with wildtype $L$. pneumophila. This protective immunity is comparable to that induced by immunization with a sublethal dose of wild-type L. pneumophila.

The induction of protective immunity by immunization with mutant L. pneumophila is paralleled by the development of both humoral and cell-mediated immune responses. Immunized but not control animals develop high titers of antibody against wild-type L. pneumophila antigens, and strong cutaneous delayed-type hypersensitivity and lymphocyte proliferative responses to wild-type $L$. pneumophila antigens.

Guinea pigs immunized twice with mutant L. pneumophila did not develop significantly greater humoral or cell-mediated immune responses to wild-type $L$. pneumophila antigens than guinea pigs immunized once. Consistent with a role for these immune responses in host defense, guinea pigs immunized twice did not exhibit significantly greater protective immunity to lethal aerosol challenge with wild-type L. pneumophila than guinea pigs immunized once.

Immunization with mutant L. pneumophila induces strong protective immunity in guinea pigs against an extremely high challenge dose of aerosolized wild-type L. pneumophila. This 
dose is much higher than that likely to be encountered by humans in nature. In view of this, the fact that the mutant is nonlethal to guinea pigs even at doses 20 times greater than the $\mathrm{LD}_{100}$ of wild-type $L$. pneumophila, and the fact that the mutant remains avirulent after guinea pig passage, the mutant has potential as a prototype vaccine against Legionnaires' disease in humans.

The concept of a live avirulent bacterial vaccine against a human intracellular pathogen has ample precedent. BCG, an attenuated strain of Mycobacterium bovis, has been used extensively in Europe and elsewhere as a vaccine against human tuberculosis.

A safe and effective vaccine against Legionnaires' disease might be particularly valuable for persons who are in high-risk groups for this disease, e.g. cigarette smokers, persons who abuse alcohol, persons with advanced malignancy, and persons on immunosuppressive therapy, especially organ transplant recipients.

\section{Acknowledgments}

We are grateful to Ms. Debora Gloria and Ms. Barbara Jane Dillon for expert technical assistance.

This work was supported by grant AI-22421 from the National Institutes of Health. Dr. Horwitz is a Gordon MacDonald Scholar at University of California, Los Angeles.

\section{References}

1. Horwitz, M. A., and S. C. Silverstein. 1980. Legionnaires' disease bacterium (Legionella pneumophila) multiplies intracellularly in human monocytes. J. Clin. Invest. 66:441-450.

2. Horwitz, M. A., and S. C. Silverstein. 1981. Interaction of the Legionnaires' disease bacterium (Legionella pneumophila) with human phagocytes. I. L. pneumophila resists killing by polymorphonuclear leukocytes, antibody, and complement. J. Exp. Med. 153:386-397.

3. Horwitz, M. A., and S. C. Silverstein. 1981. Interaction of the Legionnaires' disease bacterium (Legionella pneumophila) with human phagocytes. II. Antibody promotes binding of $L$. pneumophila to monocytes but does not inhibit intracellular multiplication. J. Exp. Med. 153:398-406.
4. Horwitz, M. A. 1983. Cell-mediated immunity in Legionnaires' disease. J. Clin. Invest. 71:1686-1697.

5. Horwitz, M. A., and S. C. Silverstein. 1981. Activated human monocytes inhibit the intracellular multiplication of Legionnaires' disease bacteria. J. Exp. Med. 154:1618-1635.

6. Nash, T. W., D. M. Libby, and M. A. Horwitz. 1984. Interaction between the Legionnaires' disease bacterium (Legionella pneumophila) and human alveolar macrophages. Influence of antibody, lymphokines, and hydrocortisone. J. Clin. Invest. 74:771-782.

7. Davis, G. S., W. C. Winn, Jr., D. W. Gump, J. E. Crayhead, and H. N. Beaty. 1982. Legionnaires' pneumonia after aerosol exposure in guinea pigs and rats. Am. Rev. Respir. Dis. 126:1050-1057.

8. Davis, G. S., W. C. Winn, Jr., D. W. Gump, and H. N. Beaty. 1983. The kinetics of early inflammatory events during experimental pneumonia due to Legionella pneumophila in guinea pigs. J. Infect. Dis. 148:823-835.

9. Baskerville, A., R. B. Fitzgeorge, M. Broster, P. Hambleton, and P. J. Dennis. 1981. Experimental transmission of Legionnaires' disease by exposure to aerosols of Legionella pneumophila. Lancet. ii:13891390.

10. Katz, S. M., and S. Hashemi. 1982. Electron microscopic examination of the inflammatory response to Legionella pneumophila in guinea pigs. Lab. Invest. 46:24-32.

11. Berendt, R. F., H. W. Young, R. G. Allen, and G. L. Knutsen. 1980. Dose response of guinea pigs experimentally infected with aerosols of Legionella pneumophila. J. Infect. Dis. 141:186-192.

12. Breiman, R. F., and M. A. Horwitz. 1987. Guinea pigs sublethally infected with aerosolized Legionella pneumophila develop humoral and cell-mediated immune responses and are protected against lethal aerosol challenge. A model for studying host defense against lung infections caused by intracellular pathogens. J. Exp. Med. 164:799811.

13. Horwitz, M. A. 1987. Characterization of avirulent mutant Legionella pneumophila that survive but do not multiply within human monocytes. J. Exp. Med. 166:1310-1328.

14. Horwitz, M. A. 1984. Phagocytosis of the Legionnaires' disease bacterium (Legionella pneumophila) occurs by a novel mechanism: engulfment within a pseudopod coil. Cell. 36:27-33.

15. Wilkinson, H. W., B. J. Fikes, and D. D. Cruce. 1979. Indirect immunofluorescence test for serodiagnosis of Legionnaires' disease: evidence for serogroup diversity of Legionnaires' disease bacterial antigens and for multiple specificity of human antibodies. J. Clin. Microbiol. 9:379-383.

16. Gabay, J. E., and M. A. Horwitz. 1985. Isolation and characterization of the cytoplasmic and outer membranes of the Legionnaires' disease bacterium (Legionella pneumophila). J. Exp. Med. 161:409422. 